\title{
Ultrafine grain recrystallisation of a metastable- $\beta$ Ti-alloy via conventional thermomechanical processing
}

\author{
A. J. Edwards ${ }^{1,2}$, P. Vermaut ${ }^{1,2}$, I. Guillot ${ }^{3}$, F. Prima ${ }^{1}$
}

${ }^{1}$ PSL Research University, Chimie ParisTech-CNRS, Institut de Recherche de Chimie Paris, 75005 Paris, France

${ }^{2}$ Sorbonne Universities, UPMC University Paris 06, UFR926, 75005 Paris, France

${ }^{3}$ Université Paris Est, ICMPE, UMR 7182 CNRS-UPEC, 2 rue H. Dunant 94320 Thiais, France

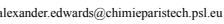

Keywords : titanium alloy, ultrafine grains, recrystallization, martensitic transformation

\begin{abstract}
$\underline{\text { Abstract }}$
Alloys with ultrafine grains (UFG) offer high strength potentially combined with ductility. Until now, producing ultrafine grains in ingot alloys has required either severe plastic deformation techniques or flash annealing, neither of which are scalable to bulk alloy production. In this work, we formed submicronic grains in the metastable $\beta$ titanium alloy Ti-20Nb-6Zr (at\%), using conventional cold rolling and annealing at $823 \mathrm{~K}$ in a conventional furnace. The cold rolling (298K) transformed the $\beta$ structure mostly to $\alpha$ " martensite, but if the rolling temperature was raised to $453 \mathrm{~K}$, martensite formation was supressed, and no grain refinement occurred during the subsequent similar annealing treatment. Therefore, we attribute the formation of ultrafine grains to a mechanism involving stress-induced martensite and its reverse transformation.
\end{abstract}

\section{Introduction}

Most strengthening approaches for improving the strength of metals do so at the expense of ductility. However, reducing grain size can improve both properties; the effect is maximized in materials with Ultra-Fine Grains (UFGs), defined as a grain size below $1 \mu \mathrm{m}$. During recrystallization, the UFGs grow from a high density of recrystallization nuclei, and this is promoted by a high density of defect-energy in the lattice. In the past few decades, Severe Plastic Deformation techniques (SPD) [1-5] have emerged as a means of attaining UFG through an unprecedentedly high density of defects. However, SPD techniques are energy-intensive, and are not viable for large-scale quantities of material.

A less-investigated alternative to producing UFGs is the Stress-Induced Martensite and Reverse Transformation (SIMRT) process. By tailoring their stability using appropriate alloying, metastable phases like martensite can be induced by straining at room temperature; this type of transformation generates a more complex stress state from plastic deformation and can potentially induce finer grain sizes after reversion.

We are aware of only two examples of SIMRT attaining UFGs using only conventional mechanical working (cold rolling): Ma and Lee et al., with a metastable austenitic steel alloy [6,7] and Cai et al. with a metastable $\beta$ titanium alloy $\left(\mathrm{Ti}_{77.47} \mathrm{~V}_{14.93} \mathrm{Al}_{6.38} \mathrm{Sn}_{1.21}\right.$ ) [8,9]. In both cases, the authors identified SIMRT as the principle cause of the observed UFG refinement.

Although both studies used conventional rolling to induce the plastic deformation instead of SPD, they followed this with flash annealing in a salt bath furnace to achieve UFG recrystallisation. Flash annealing is very effective at inducing a recrystallisation whilst minimizing grain growth: the cold worked specimen is rapidly up-quenched to the recrystallization temperature in a molten salt bath, and then quenched back to room temperature in water, after only a few minutes of annealing (Ma et al. used 10min flash annealing treatments at 937K to produce $\sim 200 \mathrm{~nm}$ grains in steel [6], and Cai et al. used 3min at $1043 \mathrm{~K}$ to produce $\sim 1.3 \mu \mathrm{m}$ grains in metastable titanium [9]). However, flash annealing is not viable when working with bulk material, thus negating the advantage of using conventional rolling for mass production.

SIMRT is not always so effective: when Sadeghpour et al. applied a similar conventional rolling and flash annealing SIMRT process to refine grains in a metastable Ti-4733 alloy [10], the finest grains they obtained were $22 \mu \mathrm{m}$; outside the range of UFGs. This was probably due to the high recrystallization temperatures they employed (e.g. 1133K) above the $\beta$-transus in their alloy and their slightly lower thickness reduction of $70 \%$ (compared to $75 \%$ by Cai et al.).

The work presented here attempts to understand the different thermomechanical and microstructural processes involved in SIMRT which induce UFGs by studying a metastable- $\beta$ titanium alloy Ti-20Nb-6Z (at\%), hereafter called TNZ. This alloy's superelastic properties and biocompatibility makes it interesting for biomedical applications [11,12], but it also has all the necessary characteristics for the SIMRT process: its $\mathrm{M}_{\mathrm{s}}$ is $246 \mathrm{~K}$ and $\alpha$ " martensite is induced by applied stress at room temperature [12]. We propose here a unique approach to isolate the role of SIMRT by comparing cold rolling, which produces stress-induced martensite (SIM), to rolling at a slightly higher temperature (453K: so-called "warm rolling”) which suppresses SIM.

\section{Experimental Procedures}

Slices of Ti-20Nb-6Zr at $\%$ were cut from a forged bar, solution treated at $1173 \mathrm{~K}$ for $15 \mathrm{~min}$ and quenched to room temperature. The solution treated samples were cut into rectangular prisms of $10 \mathrm{~mm}$ thickness for rolling. The solution treated samples were then either cold (298K) or warm (453K) rolled to a near $90 \%$ thickness reduction (starting from $10 \mathrm{~mm}$ thickness). The as-rolled samples were then annealed in a vacuum furnace by heating up at $10 \mathrm{~K} / \mathrm{min}$ up to $823 \mathrm{~K}$, maintaining $823 \mathrm{~K}$ for $30 \mathrm{~min}$ and furnace cooling to room temperature. The microstructural analysis of these samples was made by XRD and Transmission Electron Microscopy. X-ray diffraction was performed using a Bruker X-8 model using a Co X-ray source. The JEOL 2000EX TEM microscope was operated at 200kV. The thin foil

(C) The Authors, published by EDP Sciences. This is an open access article distributed under the terms of the Creative Commons Attribution License 4.0 (http://creativecommons.org/licenses/by/4.0/). 
specimens were prepared by first thinning discs of $3 \mathrm{~mm}$ diameter to $100 \mu \mathrm{m}$ by conventional grinding ( $\mathrm{SiC}$ abrasive paper) before electrolytic polishing with a 10:10:1:1 butanol - ethanol - perchloric hydrochloric acid solution at $243 \mathrm{~K}$ with $25 \mathrm{~V}$.

\section{$\underline{\text { 3. Results and Discussion }}$}

The initial solution treatment forms an equiaxed $\beta$ microstructure of $\sim 50 \mu \mathrm{m}$ grains; this is retained upon quenching to room temperature because the Ms is $246 \mathrm{~K}$ [12]. The solution treated TNZ is highly textured, with low intensity $200_{\beta}$ and $211_{\beta}$, because of the forging history of the as-received bar.

The solution-treated $\beta$ microstructure is plastically deformed by conventional rolling which causes an accumulation of dislocations and defects as is evident from the XRD peaks broadening in Figure 1.

The purpose of rolling at two different temperatures is to compare, for a relatively similar degree of plastic work, how SIM influences grain refinement. Starting from a full- $\beta$ equiaxed microstructure, cold rolling at room temperature forms predominantly stress-induced $\alpha$ " martensite as visible in Figure 1. To be able to now compare cold to warm rolling without SIM, the warm rolling temperature must be high enough to inhibit SIM, whilst at the same time low enough to avoid recovery. Warm rolling at $453 \mathrm{~K}$ was enough to inhibit $\alpha$ ": in Figure 1 we observe mostly $\beta$ with only a small martensite shoulder of $200_{\alpha "}$ whose intensity is small with respect to the $\beta$ peaks. Furthermore, the homologous temperatures for cold and warm rolling at $453 \mathrm{~K}$ are 0.15 and 0.22 respectively: with such a small difference we do not anticipate significant recovery and we anticipate there to be a comparable degree of stored elastic energy. Thus, comparing further heat treatments of these two as-rolled microstructures will allow us to isolate the role of SIM on the formation of UFGs.

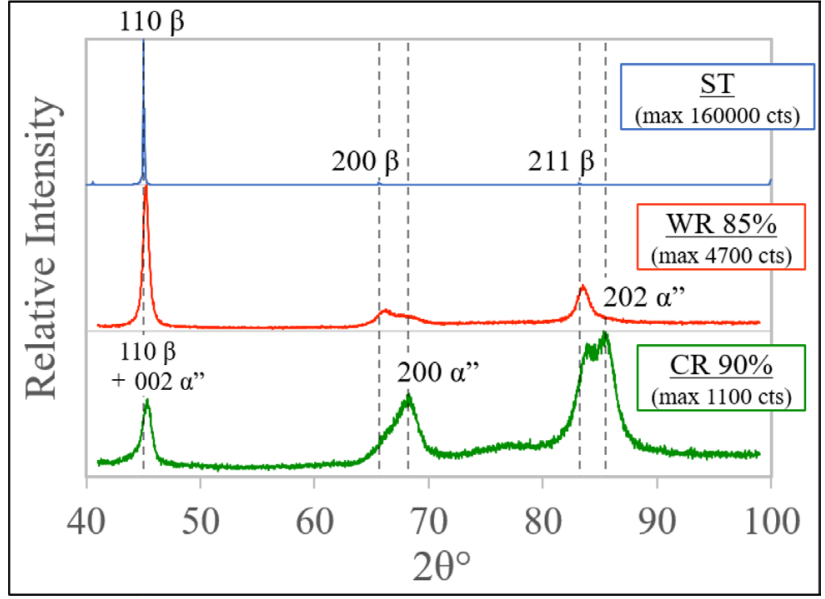

Figure 1 XRD patterns for Solution Treated (ST), 85\% Warm Rolled, $90 \%$ Cold Rolled TNZ

TNZ contains $20 \mathrm{at} \%$ niobium and $6 \mathrm{at} \%$ zirconium: these induce a lowering of the $\beta$-transus temperature to $\sim 863 \mathrm{~K}$ compared to $1156 \mathrm{~K}$ in pure Ti, so heat treatments above this temperature will form a full $\beta$ microstructure. In Figures 2 and 3 we observe that annealing at $823 \mathrm{~K}$, below the $\beta$-transus, forms two-phases $\alpha-\beta$ microstructures with very different morphologies.

In Figure 2 we observe the warm rolled TNZ after heat treatment is made up of large $\beta$ grains and precipitates of intragranular $\alpha$ platelets of $\sim 40 \mathrm{~nm}$ thickness. The selected area diffraction pattern in Figure 2 shows a large single $\beta$ grain and with no sign of subgrains. 


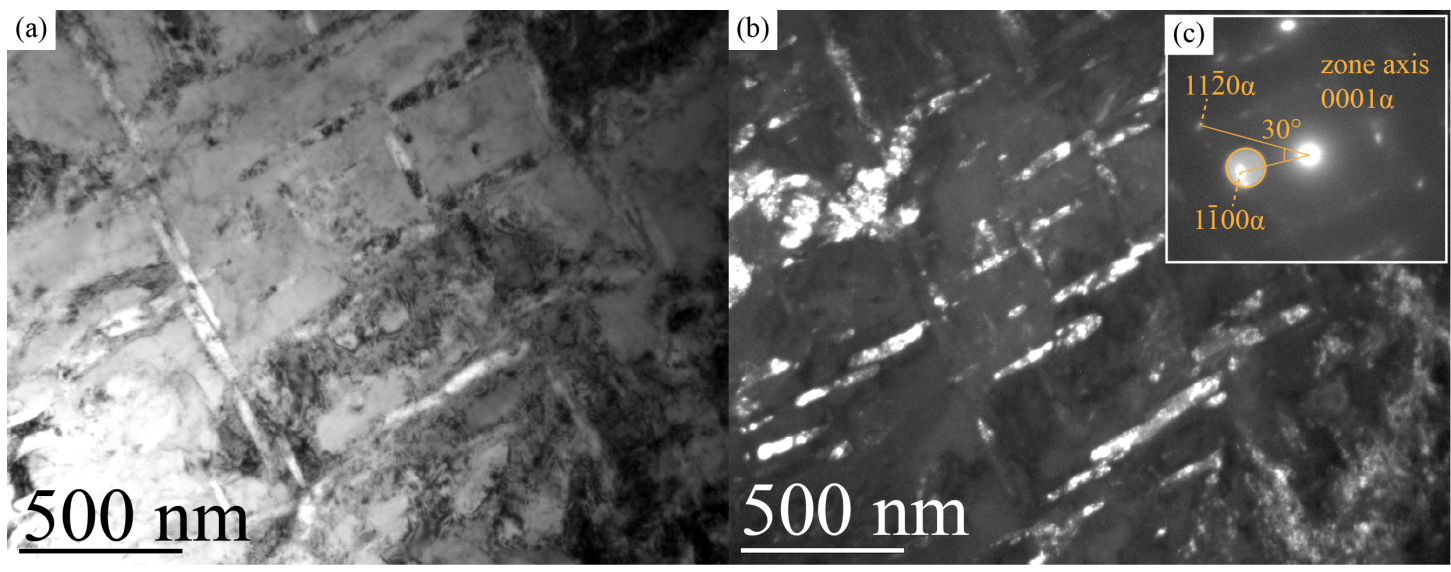

Figure 2 Warm rolled $85 \%$ TNZ annealed 30min at 823K: TEM (a) bright and (b) dark field images with (c) its corresponding selected area electron diffraction pattern. The circled electron diffraction spots correspond to the ones

selected for the dark field image.

By contrast, Figure 3 shows that after the same annealing treatment, cold rolled TNZ forms a remarkable fine-grained microstructure, consisting of globular $\sim 300 \mathrm{~nm} \beta$ and $\alpha$ grains. The selected area electron diffraction pattern in Figure 3 is predominantly made up of a ring of $110_{\beta}$ spots of $d_{110 \beta}=0.233 \mathrm{~nm}$, accompanied by a few $\alpha$ spots inside and outside of the ring. This ring-like arrangement of diffraction spots is characteristic of a polycrystalline structure of many differently oriented $\beta$ grains.

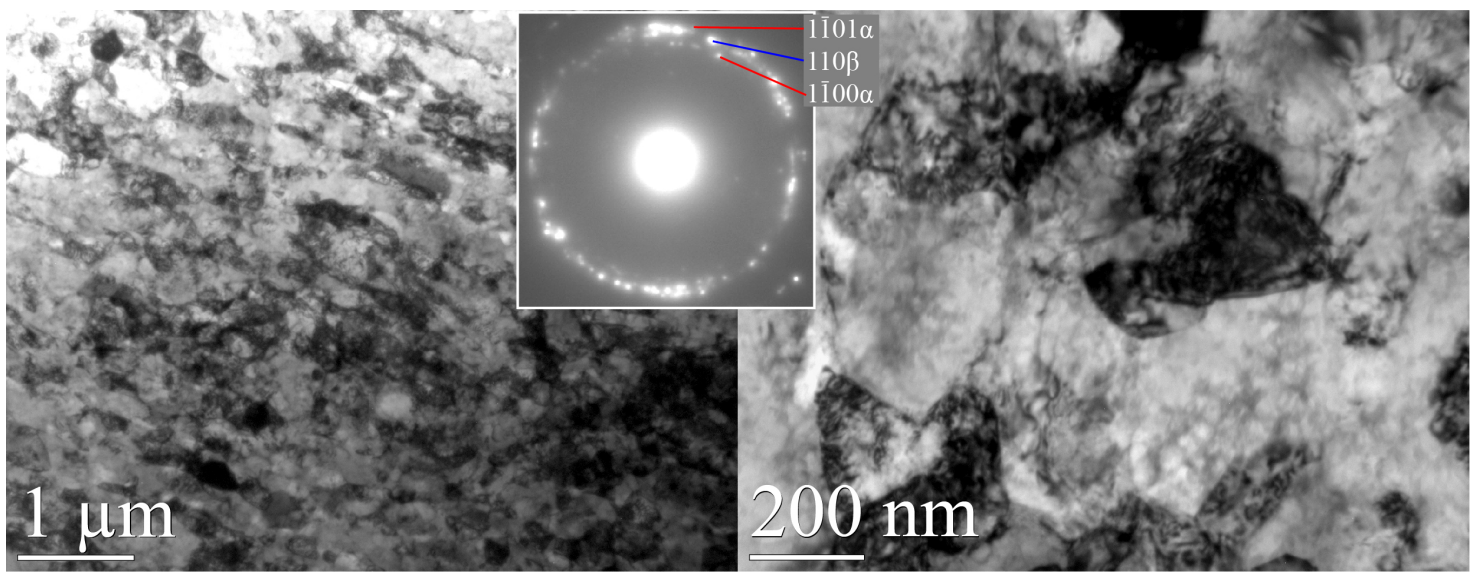


Judging from the work of Cai et al and others on SIMRT in titanium, and studies of $\alpha$ precipitation in $\beta$ titanium alloys, we may hypothesize a likely scenario that would lead to UFG refinement from cold rolled but not warm rolled TNZ.

As expected, we observed that warm rolling deforms primarily by dislocation slip, with no SIM $\alpha$ ". This means the $\beta$ grains accumulate plastic deformation through slip bands and a dense dislocation network. The equiaxed $\beta$-grains change to a pancake-shape in proportion with the change in sample shape from rolling. Normally, after cold working to store elastic energy, to induce a uniform fine grain recrystallisation it is necessary to anneal at high temperatures, at least above the $\beta$ transus (as performed by flash annealing). However, in this work we chose to use a slow continuous heat treatment at $10 \mathrm{~K} / \mathrm{min}$ up to $823 \mathrm{~K}$ to improve the scalability of the thermomechanical processing. By annealing TNZ below the $\beta$-transus in this manner, we expect recovery in combination with precipitation of first $\omega$ phase near $\sim 573 \mathrm{~K}$ and then $\alpha$ phase near $\sim 673 \mathrm{~K}[13]$.

In Figure 2 we observe intragranular $\alpha$ precipitates; Furuhara et al. and Ohyama et al. established that $\alpha$ precipitation in near $\beta$ titanium occurs on energetically favourable interfaces and sites in the microstructure such as grain boundaries, subgrain boundaries etc., before forming intragranular precipitates [14-16]. More specifically in a metastable- $\beta$ Ti-Nb-Zr-Ta alloy similar to TNZ, Nag et al. showed nanoscale $\omega$ precipitates were sites for $\alpha$ precipitation [17], this could also be possible in TNZ given our continuous heat treatment which first precipitates $\omega$ before $\alpha$. The morphology of $\alpha$ platelets in Figure 2 is like the ones observed by Nag et al. except theirs were only a few nanometres wide instead of $\sim 40 \mathrm{~nm}$. This is probably due to their lower annealing temperature of $673 \mathrm{~K}$ when diffusion is slower: Nb has limited solubility in both $\omega$ and $\alpha$ phase which leads to the partitioning of $\mathrm{Nb}$ out of the precipitates and into the surrounding $\beta$ [17]. The diffusion limiting nature of $\alpha$ precipitation limits the growth rate of $\alpha$ particles and favours the formation of thin and parallel platelets in addition to the crystallographic considerations when in a burgers orientation relationship with $\beta$.

We conclude from Figure 2 that warm rolling, without SIM, does not cause a significant $\beta$ grain refinement, but there was recovery in addition to the precipitation of intragranular $\alpha$ platelets. On the other hand, cold rolling with SIM induces a remarkable UFG refinement in TNZ (figure 3) after the same annealing at 823K. Cai et al. who also induced UFG via SIMRT attributed it to these two factors: the reversion process of $\alpha$ " to $\beta$ and the recrystallisation of the $\beta$ phase.

We know from Figure 1 that significant SIM $\alpha$ " is formed in cold rolled TNZ in addition to a high density of dislocations. Zafari et al. describes how a metastable $\beta$ titanium alloy deformed by high pressure torsion induces intersecting $\alpha$ " lathes which divide the equiaxed $\beta$ grains into much smaller $\beta$ cells. Further deformation of the initially formed $\beta$ causes this process to now repeat inside each $\beta$ cell, leading to an even finer network of $\beta$ cells demarcated by more martensite [18]. In addition to the SIM, most of the plastic deformation consists of dislocation slip which will fragment the martensite lathes even further The interaction between dislocations and the myriad of $\beta$ - $\alpha$ " interfaces induces a complex stress state. This form of nanostructuring is corroborated by observations by Cai et al in their cold rolled samples: their TEM analysis found $\alpha$ " lathes even just a few nanometers apart [8]. It is likely that a similar fragmentation mechanism of the initially large $\beta$ grains occurs during cold rolling of TNZ, which may not be possible during warm rolling where there is no SIM.

The finish temperature $\left(\mathrm{A}_{\mathrm{f}}\right.$ ) for the reversion of thermally induced $\alpha$ " martensite (from cooling below Ms) back to $\beta$ in TNZ is $267 \mathrm{~K}$ [12]. In the case of stress induced $\alpha$ " from cold rolling, even though it is not stable at room temperature, it cannot transform back to $\beta$ because of the high density of defects (dislocations) and high-level local stresses. Additional energy in the form of heat is necessary to overcome this obstruction, and therefore a higher temperature than predicted by $\mathrm{A}_{\mathrm{f}}$ will be necessary to transform to $\beta$.

It could be argued that the new equiaxed $\beta$ UFGs in figure 3 come directly from the SIM and the reversion of $\alpha$ " to $\beta$ as is observed during SPD techniques [18]. However, Cai et al. using conventional rolling and the annealing only achieved a uniform equiaxed UFG size when flash annealing was done above $993 \mathrm{~K}$; at lower temperatures, $653 \mathrm{~K}$ and $853 \mathrm{~K}$, nanosized $\alpha$ " martensite and $\alpha$ precipitates were observed but not equiaxed $\beta$ grains [8]. They concluded that recrystallisation appears to be a necessary component of UFG refinement by SIMRT when cold rolling and annealing.

In TNZ, a remarkable low temperature recrystallisation of equiaxed UFG $\beta$ has been obtained after annealing at only $823 \mathrm{~K}$ (figure 3 ). It is possible that this is in part due to the higher stability of $\beta$ phase in TNZ which has a lower $\beta$ transus temperature, and the higher reduction in thickness in the rolling (75 to 90\%). However, we showed that warm rolled TNZ, without SIM, and heating up at the same $10 \mathrm{~K} / \mathrm{min}$ to $823 \mathrm{~K}$ only induced recovery of large $\beta$ grains, therefore showing that SIM is a necessary condition to recrystallization at so low a temperature. The question now is where does the additional driving force for recrystallisation at such a low temperature in cold rolled TNZ come from?

Upon heating, $\alpha$ " would like to reverse to $\beta$ phase. This should proceed by a fully displacive mechanism. However, the local stress due to the high density of dislocations strongly stabilizes the martensite and prevents the formation and glide of $\alpha " / \beta$ interfaces which is necessary for the reverse transformation. The reverse transformation will then tend to occur at much higher temperature, far from the equilibrium temperature between the two phases. Since the reverse transformation is occurring at a much higher temperature, the chemical driving force associated with this reversion will also be much higher.

One can also expect that the martensite, in addition to the high density of dislocation lines, contains a high density of interfaces separating the different martensite variants. Such interfaces can constitute obstacles for the dislocation lines; this would delay the recovery mechanism which will then shift to higher temperatures. In addition to the martensite being retained to higher temperatures, it will also undergo only limited recovery and therefore keep in its lattice a high density of elastic energy.

Recrystallization is a diffusional transformation which proceeds by nucleation and growth, and its driving force corresponds mainly to the elastic energy stored in the lattice defects. In the case of warm rolled samples, the $\beta$ phase has time during the slow heating to recover, preventing recrystallization because of the strong decrease of available elastic energy. In the case of cold rolling, the recovery of the deformed $\alpha$ " is probably more difficult and shifted to higher temperatures. The recrystallization process will then be easier because it will combine two driving forces: firstly, a high density of elastic energy which remains available because of slow recovery, and secondly, the reversion of $\alpha$ " to $\beta$, because the $\alpha$ " is likely to persist to higher temperature. It is expected that the sum of these two driving forces enables the formation of a high density of new nuclei at a relatively low temperature for recrystallisation. Because the temperature at which recrystallization starts is below the $\beta$ transus temperature, both alpha and beta phases can nucleate, leading to the UFG microstructure we observed. 
The flash annealing treatments from previous works had to be very short to avoid grain growth. On the other hand, the low temperature recrystallisation in TNZ retains its fine grain size even after 30 minutes at $823 \mathrm{~K}$ because grain growth is slower at lower temperature and $\alpha$ phase will precipitate and pin the grain boundaries at the onset of recrystallisation.

\section{Conclusions}

After annealing at $823 \mathrm{~K}$, TNZ cold-rolled to $90 \%$ produced ultra-fine $\alpha$ and $\beta$ grains with $\sim 300 \mathrm{~nm}$ diameter, whereas it is completely absent if the rolling was carried out at $453 \mathrm{~K}$, which supresses straininduced martensite. We attribute such grain refinement to the combination of:

- stress-induced martensite and its reverse transformation (SIMRT),

- a relatively low $\mathrm{A}_{\mathrm{f}}$ temperature which provides an important chemical contribution to the recrystallization driving force from the reversion of $\alpha$ " to $\beta$

- a relatively low $\beta$-transus temperature allowing formation of both $\alpha$ and $\beta$ phase during recrystallisation, which prevents grain growth when the recrystallisation temperature is maintained.

As far as we are aware, this is the first report of SIMRT producing UFG in any alloy using both conventional working and slow heating/cooling rates which are feasible at industrial scale.

\section{References}

[1] R. Z. Valiev, A. V. Korznikov \& R. R. Mulyukov. Mater. Sci. Eng. A 168 (1993) 141-148

[2] R. Z. Valiev, R. K. Islamgaliev \& I. V. Alexandrov. Prog. Mater. Sci. 45 (2000) 103-189

[3] W. Yinmin, C. Mingwei, Z. Fenghua \& M. En. Nature 419 (2002) 912-914

[4] V. V. Stolyarov, Y. T. Zhu, T. C. Lowe, R. K. Islamgaliev \& R. Z. Valiev. Nanostructured Mater. 11 (1999) 947-954

[5] R. Z. Valiev, A. V. Sergueeva \& A. K. Mukherjee. Scr. Mater. 49 (2003) 669-674

[6] Y. Ma, J. E. Jin \& Y. K. Lee. Scr. Mater. 52 (2005) 1311-1315

[7] Y. K. Lee, J. E. Jin \& Y. Q. Ma. Scr. Mater. 57 (2007) 707-710

[8] M. H. Cai, C. Y. Lee, S. Kang \& Y. K. Lee. Scr. Mater. 64 (2011) 1098-1101

[9] M. H. Cai, C. Y. Lee \& Y. K. Lee. Scr. Mater. 66 (2012) 606-609

[10] S. Sadeghpour et al. Mater. Sci. Eng. A 731 (2018) $465-478$

[11] F. Sun, et al. Scr. Mater. 63 (2010) 1053-1056

[12] F. Sun, et al. J. Mech. Behav. Biomed. Mater. 4 (2011) 1864-1872

[13] D. L. Moffat \& D. C. Larbalestier. Metall. Trans. A 19 (1988) 1687-1694

[14] H. Ohyama, H. Nakamori, Y. Ashida \& T. Maki. ISIJ Int. 32 (1992) 222-231

[15] T. Furuhara, T. Maki \& T. Makino. Mater. Japan 117 (2001) 318-323

[16] T. Furuhara, S. Takagi, H. Watanabe \& T. Maki. Metall. Mater. Trans. A 27A (1996) 1635-1646

[17] S. Nag, R. Banerjee \& H. L. Fraser. J. Mater. Sci. 44 (2009) 808-815

[18] A. Zafari, X. S. Wei, W. Xu \& K. Xia. Acta Mater. 97 (2015) 146-155 